\title{
Implementation of a positive psychology group program in an inpatient eating
}

disorder service. A pilot Study

Enrique, Ángel ${ }^{\mathrm{a}}$ (enriquea@tcd.ie), Bretón-López, Juana ${ }^{\mathrm{b}, \mathrm{c}}$ (breton@uji.es), Molinari, Guadalupe $^{\mathrm{b}}$ (molinari@uji.es); Llorca, Ginés ${ }^{\mathrm{d}}$ (ginesllorca@ hotmail.com) and Botella, Cristina $^{\mathrm{b}, \mathrm{c}}$ (botella@uji.es)

${ }^{a}$ E-mental Health Research Group, School of Psychology, University of Dublin, Trinity College, Dublin.

b Department of Basic Psychology, Clinic and Psychobiology, University Jaume I, Castellón, Spain

c CIBER Fisiopatología Obesidad y Nutrición (CIBERobn), Instituto Salud Carlos III, Spain

d Psychiatry Service, Consorcio Hospitalario Provincial de Castellón, Castellón, Spain

\section{Corresponding author at}

e-mail address: enriquea@tcd.ie (Angel Enrique).

Conflict of Interest: The authors declare that they have no conflict of interest.

How to cite: Enrique, A., Bretón-López, J., Molinari, G., Llorca, G., \& Botella, C. (2020). Implementation of a positive psychology group program in an inpatient eating disorders service: a pilot study. Eating and Weight Disorders-Studies on Anorexia, Bulimia and Obesity, 1-10. Doi: https://doi.org/10.1007/s40519-020-00908-5 


\title{
Implementation of a positive psychology group program in an inpatient eating disorder service. A pilot Study
}

\begin{abstract}
Context: Eating disorders (ED) are very difficult conditions to treat. Therefore, interventions in this field are shifting their main target towards the disorder's impact in quality of life, rather than ED symptomatology in itself. In this sense, a focus in the promotion of positive emotions and well-being is emerging in order to ameliorate the harmful effects caused by ED. However, evidence of potential benefits of this type of interventions is still scarce.
\end{abstract}

Purpose: This study introduces a 4-week positive psychology group program specifically designed for ED patients' needs and to present data about feasibility and acceptability.

Method: Seven female inpatients of an eating disorder service aged from 13 to 38 years old attended the group. Measures of affect and optimistic thinking were taken before the program and after each session.

Results: The program was very well rated by participants, and there was no attrition. Furthermore, possible benefits were found in terms of optimistic thinking at the end of the group program, and these benefits were noticeable, but non-significant, in the case of affect.

Conclusions: This study opens the door to conduct larger and controlled studies for testing interventions aimed at promoting positive emotions and well-being in ED populations. Thus, these interventions could support the efficacy of current treatments in order to improve patients' quality of life.

Keywords: eating disorders, positive psychology, affect, optimistic thinking, anorexia nervosa

Level of evidence: Level IV, case series 


\section{Introduction}

Eating Disorders (ED), including anorexia nervosa (AN), bulimia nervosa (BN), and nonspecified eating disorders (EDNOS), are considered an important cause of physical and psychosocial morbidity and mortality in young individuals [1]. Cognitive-behavioral therapy for bulimia nervosa has been found effective and it is the recommended treatment for bulimia nervosa [2]. However, there is no empirical evidence to support the use of one treatment over another for AN and EDNOS patients [3]. Due to the difficulty in treating these conditions, epidemiological studies have shown that many patients follow a severe and enduring course and at least one-third of patients attending therapy drop out [4].

Furthermore, the burden of the disease causes impairment in many areas, beyond the eating disorders symptomatology, such as social, psychological and physical areas, producing a strong impact on their quality of life [5]. It has been found that several eating disorder-related symptoms, such as extreme worry about weight, body dissatisfaction, and exercising to lose weight, are negatively related to life satisfaction [6]. In this sense, Kitsantas and colleagues [7] found that individuals with eating disorders reported lower levels of positive affect and higher levels of negative affect compared to unaffected peers.

Given the broad impairment and the limited treatment efficacy of treatments, some authors maintain that the main target of the treatments should be the disorder's impact on quality of life, rather than the ED symptomatology itself [4]. This approach, along with patients' perspectives about recovery, claims that recovery is more than just the absence of ED symptoms, and it focuses on the need of promoting positive functioning aspects [8]. Following this vein, factors such as optimism, self-determination, self-esteem, and satisfaction with life buffer the harmful effects of body dissatisfaction on women with bulimic symptomatology [6]. Thus, these authors claimed that, in women who are satisfied with their lives, feel good about themselves, and are optimistic about the future, 
body dissatisfaction may be less impairing in determining their experiences, goals, values, and feelings about their self. Despite these findings, the inclusion of interventions for patients with ED aimed at improving positive emotions and well-being is still very recent and scarce. Given the potential benefits of these emotions, Tchanturia and colleagues [9] suggested that the introduction of Positive Psychological Interventions (PPIs) could play a role in the recovery of patients with EDs. PPIs could be of help by promoting the emergence of positive emotions and problem-solving strategies related to their health behaviors. Moreover, Tchanturia [9] stated that the inclusion of PPIs in ED prevention could enrich treatment programs and even enhance their impact. To our knowledge, only one pilot study has tested the feasibility of a 5-session PPI group program in an adolescent ED inpatient service [10]. The program was composed of a combination of PPIs (i.e. three good things, gratitude letter, acts of kindness, loving-kindness meditation, and identifying strengths), showing potential benefits in majority of participants in terms of life satisfaction and subjective happiness, and the attrition rate was zero.

The selection and combination of PPIs in order to develop tailored treatments for specific conditions should account for the needs of the patients, the features of the PPIs and the fit between both [11]. In this sense, it has been shown that patients with ED often view recovery as unimaginable because they are not able to envision a better personal future [12]. However, variables such as optimism, goal setting and expectations about the future are crucial for recovery in patients with ED and they play a role in their engagement with the treatment [13]. For this reason, Malson et al. [12] stated that strategies aimed to generate alternative self-constructions that challenge the essential bulimic/anorexic self might be quite useful for recovery. Thus, improving optimistic thinking in patients with ED might have a strong impact on reducing potential negative effects of eating symptomatology. In this regard, one PPI that has been found to be very useful in 
improving positive affect and optimistic thinking is the Best Possible Self exercise (BPS; for a review, see [14]. BPS consists of writing about and envisioning a future where everything has turned out in the best possible way in different life domains [15]. This study proposes an adaptation of the BPS exercise that can help to construct a new prospective self, beyond the anorexic or bulimic one. Given that this is a first approach to the inclusion of PPIs in clinical settings, along with the pilot study carried out by Harrison and colleagues [10], other pilot studies are needed in order to generate pilot data. These studies allow to develop consistent practices and protect human subjects from potential harmful effects, which are crucial factors before developing larger trials [16].

In sum, this pilot study was primarily aimed to test the feasibility and potential benefits of implementing a 4-week positive psychology group intervention focused on improving positive affect and optimistic thinking, in inpatients of a public mental health service for individuals with eating disorders. Given the benefits that these interventions have produced in prior studies [10], it was hypothesized that the intervention would produce significant improvements in affect and optimistic thinking after the intervention. Furthermore, a second goal was to test the feasibility and acceptability of the intervention. As previous studies have suggested these interventions are well accepted by patients with ED [9, 10], it was hypothesized that inpatients would report high levels of satisfaction and that dropout rates would be very low.

\section{Methods}

\section{Participants}

The sample was composed of 7 female patients. Five patients were diagnosed with anorexia nervosa ( 3 restrictive-type AN and 2 purging-type AN), and two had a diagnosis of non-specified ED (EDNOS). The two patients diagnosed of EDNOS had a prior 
diagnosis of anorexia nervosa (one restricting and the other purging subtype) 3 months before the study began, but a change in some of the symptoms before the start produced that they did not fit within the specific category anymore and therefore a diagnosis of EDNOS was given. All established diagnoses were based on the revised version of the Diagnostic and Statistical Manual of Mental Disorders, fourth edition (DSM-IV-TR) [17]. All participants were inpatients of the ED service of a public hospital in Castellón (Spain). The ages of the patients ranged from 13 to 38 years old $($ Mean $=22,8, \mathrm{SD}=11,85)$. Table 1 describes sociodemographic and clinical characteristics of the patients involved in the trial.

\section{INSERT TABLE 1}

\section{Positive Technology Application}

Book of Life. This is a module of the Earth of Well-being system, which is a computerized program composed of different activity modules. Each module is targeted to promote specific positive emotions (such as relaxation or joy) through emotional induction procedures implemented in the system. As a whole, it has shown efficacy in promoting positive emotions $[18,19]$. Specifically for this study, only a specific module called "Book of Life" was selected. This module looks like a personal diary with different chapters, each with its own instructions, in order to write about different topics. In addition, the essay can be supported with multimedia content. In this case, one of the chapters was adapted by removing the instructions, since they were provided by the therapist. Thus, the program allows pictures, music and videos to be added, either selected from the system or introduced by the user (adding their own pictures, music and videos). Hence, it is considered a flexible tool and can be used in different contexts [18, 19].

\section{Design}


A longitudinal design was employed to explore potential differences in affect and optimistic thinking throughout the 4-week program, comparing the scores at preassessment to the scores on these measures after each session and at the end of the group intervention program. Furthermore, the study was approved by the Ethics Committee of the Castellón Provincial Hospital.

\section{Measures}

Feasibility and acceptability measures

Subjective Probability Task (SPT) [20]. This scale measures positive and negative expectations about possible events that might happen in the future. It is composed of 30 items, 20 related to negative expectations that can occur in the future (e.g. "Other people won't like you "), and the other 10 focused on positive expectations (e.g. You'll have a lot of good moments with friends). The items are rated on a 7-point Likert scale, where participants are asked to estimate the likelihood of each situation happening to them in the future, from 1 (not at all likely to occur) to 7 (extremely likely to occur). Higher scores on the different subscales represent a higher perceived likelihood of the occurrence of positive and negative events in the future. It has shown appropriate levels of internal consistency for positive and negative expectations ( $\alpha=0.82$ and 0.91 , respectively; [21]. In a sample of eating disorders patients, Chronbach's alpha were .86 for positive future expectations and .94 for negative future expectations, showing excellent psychometric properties [22].

Positive and Negative Affect Scale (PANAS; [23]. To assess affect, a Spanish adaptation of the PANAS was used [24]. This scale is composed of 20 items: 10 items measuring positive affect states and 10 items measuring negative affect states. The items are rated on a 5-point Likert scale ranging from 1 (not at all) to 5 (extremely). PANAS is one of 
the most widely used instruments to measure affect because it shows excellent psychometric properties (Cronbach's alphas from 0.87-0.91). In a sample of eating disorders patients, Chronbach's alpha were .89 for positive affect and .89 for negative affect [22].

Credibility/ Expectancy Questionnaire [25]. This questionnaire was adapted from Borkovec and Nau's instrument for assessing patients' opinions and acceptability ratings of the treatment at the end of the final session. Thus, they were asked to indicate on a 10point Likert scale from 0 (not at all) to 10 (very much), their degree of satisfaction, the usefulness of the program as a whole, and whether they would recommend it to a friend. Moreover, they were asked about their levels of satisfaction with and usefulness of the technology implemented, the Book of Life, which is described above. The questionnaire also included open questions about the perceived benefits and troubles with the intervention.

\section{Additional Measures}

Eating Attitudes Test (EAT-26) [26]. This questionnaire assesses a broad range of attitudes and behaviors related to anorexia nervosa. It is composed of 26 items rated on a 6-point Likert scale from 0 (never) to 6 (always), based on the frequency with which the individual carries out the behavior or has the thought described by the item. The instrument has shown excellent psychometric properties [27, 28].

Imagen [29]. This questionnaire assesses body dissatisfaction, which means feelings of discomfort about one's body image. It is composed of 37 items rated on a 5-point Likert scale from 0 (almost never/ never) to 4 (almost always/ always). The instrument has shown adequate psychometric properties in a clinical population for the total score and each subscale (Cronbach's alphas from 0.87-0.97) [29]. 


\section{Procedure}

The group program took place in the ED service. It was carried out by one of the authors (AE), who has postgraduate studies in Eating Disorders. The program was called "My Best Self' because it was based on the development of an ideal self and the ways to reach it. It was composed of 4 sessions (one per week) lasting 2 hours each. Before the program, all the patients signed an informed consent form, and in the case of under-age patients, this consent form was signed by their parents. After that, participants completed two measures of feasibility, PANAS and SPT, along with additional measures, EAT-26 and IMAGEN, to assess severity of the ED symptomology. They were asked to deliver these questionnaires to the nurses of the clinical service before starting the first session. The sessions were all conducted in the same room and by the same therapist, who supported the content of each session with a PowerPoint presentation. Participants sat in a circle around the therapist. At the end of each session, participants filled out the PANAS and SPT measures. In addition, as a homework task, patients were asked to write the essay in the Book of Life system, and they were invited to visualize the content of the essay every day for at least 5 minutes. Patients were provided with a unique username and password to $\log$ on to the virtual platform during their free time in the service. Once the four-week program was over, patients were asked to complete the post-assessment, which included acceptability and feasibility measures. Once the post-assessment was completed, each participant received a $\mathrm{CD}$ with a video of the content developed in the system, and they were invited to continue practicing the exercise to the extent that it would be useful for them.

\section{Group intervention development}

The use of the visualization of a best possible self as a central exercise was based on prior literature that indicates the effectiveness of this exercise in improving optimism [14]. 
Moreover, given that ED patients find it difficult to imagine a better future, we included components of the hope theory [30] to facilitate the exploration of ways to reach these goals (pathways thinking) and the persistence to get them (agency thinking). In addition, we expected that sharing goals with peers would help patients to gain more insight into their own goals and enhance their engagement with reaching them. The work group was led by an external clinician who also paid attention to the content of the essay, making sure that patients did not develop damaging prospective selves in terms of their ED.

\section{Setting and context}

The group intervention emerged as a supportive tool for promoting optimism and positive affect in a forced environment (inpatient service), where patients usually lose external reinforcements. The 4 group sessions were implemented in a 2-hour, weekly format. This program took place during the morning, and it was presented to patients as a complementary workshop to the activities that they usually did in the service. For this reason, all the inpatients who were receiving ongoing treatment in the service were invited to participate in the group intervention. Attendance was voluntary, and they were told that they could drop out if they did not feel comfortable or if they found it boring.

\section{Treatment as usual}

The ED service is a day hospital that offers high intensity treatment for patients with severe presentations of an eating disorder. The treatment takes a multidisciplinary approach by considering biological, psychological, familial and sociocultural aspects. The specific goals of the service are as follows:

a) To restore healthy food habits;

b) To restore and normalize weight and nutritional status;

c) To offer control measures to prevent compensatory behaviors; 
d) To eliminate dysfunctional behavior patterns;

e) To involve patients and relatives as active collaborators in the treatment process;

f) To identify and address psychopathological symptoms associated with eating disorders.

According to these goals, different therapeutic interventions are carried out at the ED service, which comprise different tasks executed by different clinicians:

a) Three first intakes of the day (breakfast, brunch and lunch) are conducted with supervision and modelled by service staff;

b) Weight check and nutritional advice conducted by clinical nurses;

c) Individual sessions of CBT with a clinical psychologist;

d) Weekly visit with the psychiatrist to check physical status, medical complications and to prescribe medication;

e) Weekly family visits to improve conflicts, communication and relationships between patients and their relatives;

f) Group therapy with a nutritionist;

g) Workshops aimed at: disorder awareness, social skills, self-esteem, body image, relaxation, manual arts and reading.

\section{Description of the group intervention}

The content of each session is described in Table 2. Session 1 and 2 introduced the psychoeducation and program rationale to the patients, along with the instructions for the development of the ideal self and the ways to reach it. Session 3 was focused on reviewing all the content introduced in prior sessions and possible difficulties associated with the exercise. Last session was used as a summary of the program as a whole and the importance of setting life goals according to values was also remarked. 


\section{INSERT TABLE 2}

\section{Data Analysis}

Given the nature of a pilot study, with a small sample size, non-parametric analyses were used to explore potential benefits. Pre-post non-parametric Wilcoxon Signed-rank analyses were performed to explore changes in affect and future expectations. Nonparametric Mann-Whitney tests were conducted to explore if the level of change in affect and future expectations (post minus pre) was different between participants aged below and above 18 years old. Furthermore, the pre-post change was also explored using reliable change scores (RC) that were calculated for each patient for the two outcome measures, PANAS and SPT. The equation used for this analysis was extracted from Jacobson and Truax's guidelines [31]. Thus, to show reliable improvement, a RC score equal to or above a predetermined cut-off is needed. This cut-off is established by subtracting the standard deviation of a reference group on the outcome measure from their group mean and dividing this score by 2 [31]. The data used to calculate the cut-off for SPT were taken from the Spanish validation of this instrument in a clinical population[32]. In the case of PANAS, we used the spanish validation in general population, given there is no validation with clinical samples [24]. Lastly, in order to explore the evolution of the scores over time, a non-parametric Friedman's test for multiple time points was used.

\section{Results}

Scores on the EAT-26 ranged from 15 to 64 , and the average score was $31.6(\mathrm{SD}=16.75)$, indicating the presence of ED symptoms because it was above the predetermined cut-off score of 20 [27, 28]. Only one patient scored below 20. Regarding the bodydissatisfaction scale (IMAGEN), the average of the total scores was $79.71(\mathrm{SD}=26.04)$, and patients were classified according to severity degrees of body-dissatisfaction, based 
on women at risk of eating pathology [29]. Thus, $14.28 \%$ were considered severe, $42.86 \%$ moderate, and $42.8 \%$ mild in terms of body-dissatisfaction.

\section{Dropout}

Regarding session attendance, all seven patients attended sessions 1 and 3, six attended session 2, and five attended session 4. All the non-attendance cases were due to personal problems that kept them from attending the specific session. There are three main reasons why patients would not attend a specific session: 1) visit to other health specialists; 2) inpatient with partial attendance to the service (those who attend half of the week); 3) non-attendance without prior notification. Despite the non-attendance, all the patients completed the questionnaires at the different time points.

\section{Possible benefit derived from the group}

Table 3 shows the results obtained by the participants before and after the group program on the outcome measures, namely PANAS and SPT.

\section{INSERT TABLE 3}

Results of the related-samples Wilcoxon signed-rank tests showed a significant improvement on both subscales of the SPT measure, positive and negative. In the case of the two PANAS subscales, there was an improvement, but without reaching statistical significance.

Mann-Whitney U (MWU) tests were conducted to explore if the level of improvement in PANAS and SPT was different between patients below and above 18 years old. Results did not show significant differences between age groups for any of the four outcomes (SPT-POS, MWU=4.0, $p=.46 ;$ SPT-NEG, MWU=6.0, $p=1.00 ;$ PANAS-POS, $\mathrm{MWU}=3.0, p=.28 ;$ PANAS-NEG, $\mathrm{MWU}=4.0, p=.48$ ). 
The reliable change index analyses indicated that for positive future expectations, $43 \%$ of the participants reported a reliable clinical change in this outcome. In terms of negative expectations, these analyses showed that $85.7 \%$ of the participants also experienced a meaningful change in this subscale. Regarding positive affect, $28.5 \%$ of the participants reached a reliable change, and for negative affect, $43 \%$ of the sample showed a reliable change.

\section{Evolution of the scores over time}

Figures 1 and 2 display the scores obtained by the participants on the different outcome measures across the different time frames. For the comparison between moments, Friedman's test was performed for each measure.

Regarding the future expectations measure, results showed an improvement from baseline to the first session, and then the results were maintained in the following sessions. In terms of positive expectations, Friedman's test showed a trend toward significance $\left(\chi^{2}=\right.$ 9.13; $p=0.058$ ), indicating a change in the scores in the different time frames. With regard to negative expectations, Friedman's test showed a significant change between time assessments $\left(\chi^{2}=11.26 ; p=0.024\right)$. In the case of affect, there was an observable trend toward improvement from baseline on both subscales across the four sessions. In terms of positive affect, Friedman's Test did not show a significant change between time assessments $\left(\chi^{2}=5.74 ; p=0.22\right)$, in contrast to negative affect, which showed a trend towards significance $\left(\chi^{2}=9.38 ; p=0.052\right)$.

\section{INSERT FIGURE 1}

Figure 1. Evolution of positive and negative future expectations over time

Footnote: SPT: Subjective Probability Task; SPT-POS: Subjective Probability TaskPositive expectancies; SPT-NEG: Subjective Probability Task - Negative expectancies 
Figure 2. Evolution of positive and negative affect over time

Footnote: PANAS-POS: Positive and Negative Affect Scale- Positive Affect; PANASNEG: Positive and Negative Affect Scale- Negative Affect

\section{Acceptability}

The Credibility/Expectancy questionnaire was administered to measure the patients' ratings of the positive psychology group program. Results showed moderately high levels of satisfaction $(M=7.5, S D=2.07)$, usefulness $(M=8.5, S D=1.05)$, and recommendation of the program $(M=8.5, S D=1.47)$, suggesting high acceptance of the program by the patients. Regarding the positive technology used, the Book of Life, participants reported good levels of satisfaction $(M=8.6, S D=1.14)$ and usefulness $(M=8.8, S D=1.3)$, suggesting that they valued the supporting role of the technology in performing the exercise.

Regarding qualitative feedback, some participants left comments about the benefits and difficulties found in doing the exercise and with the group. Thus, some benefits reported by the patients were 'Now I have my goals clearer and I've learned to be patient with long-term goals' (P.4), 'Being able to imagine an ideal self and feeling that I can reach my own goals' (P.2) or 'now I am more aware of my goals' (P.5). Regarding difficulties, two participants found tiresome completing the questionnaires after each session (P.3 and p.6), one felt that some sessions were too long and boring (P.4), another did not like having to do the howework on the computer (P.7), and one participant reported 'facing my actual self and being aware of how far I am from my ideal self' (P.2).

\section{Discussion}

This pilot study presented the development of a four-session group intervention aimed at improving affect and optimistic thinking in ED inpatients who were being treated in a 
specialized mental health service (day hospital). These effects were explored after each session and at the end of the 4-week program, along with the levels of acceptance and usefulness of the group program and the technology implemented.

Results showed that there was no dropout at the end of the program and all patients remained in the group until the last session. This is consistent with the previous pilot study, which also found no dropouts [10], suggesting that patients usually show good adherence to these group programs that include PPIs.

Regarding the possible benefits derived from the program, there was a significant decrease in negative future expectations across the intervention period, and this change showed a trend toward significance in the case of positive expectations. The change in affect, on both the positive and negative subscales, did not reach significance, although it was noticeable at a clinical level. Despite of the broad age range, no differences were found between age groups for any of the four outcomes. Most of the patients obtained a reliable change in negative expectations, but these percentages decreased to less than half of the sample in the case of positive expectations. The difference between these percentages might be explained by the fact that those patients who did not reach a reliable change in positive expectations started with higher scores on positive expectations, and so there could have been a ceiling effect. In regards to affect, the exercise seemed less effective in improving these variables, which would explain the lower rates of meaningful change. These results suggest that this group program, focused on the future in terms of values and goals, was able to improve optimistic thinking in patients who usually have difficulties in visualizing a better future [12]. Furthermore, the changes in affect, even though not entirely significant, suggest that affect may also be improved in patients who usually have this area affected [33]. 
In terms of acceptability, the data collected suggest that the group intervention and the technology used was well received by patients. High rates of treatment acceptability reported by patients are important in order to enhance the effectiveness of interventions [34]. With regard to the qualitative feedback, patients reported that focusing on their goals and thinking about ways to reach them was not only useful in clarifying their goals, but also for allowing them to feel that these goals were attainable. In this sense, it is suggested that the presence of hope in patients with mental illness could enhance their motivation to engage in the recovery process [35]. Also, one patient reported feeling far from her ideal self. In these cases, it is important to highlight that setting goals based on our values is the only way to make sure that the path toward the goals will be meaningful in itself [36]. Furthermore, another important way to deal with these kinds of feelings when implementing exercises focused on life goals, like the BPS, is to make clear that we do not reach all our life goals, as these goals sometimes change over time or need to be adjusted, and individuals have to be prepared for this [37].

The introduction of positive technology to support the development of their best self was positively rated, since patients could accompany their essays with their own pictures and music, enhancing their ability to become immersed in the visualization [38].

Regarding the study limitations, it is important to note that, given the small sample size, the results derived from the implementation of the exercise can hardly be generalized to other populations [16], although they can be considered when designing novel interventions focused on improving quality of life and well-being. Another important limitation was the lack of a control group because it keeps us from attributing the possible benefits to the group program, instead of to other uncontrollable factors, such as the ongoing treatment they were receiving. However, the clinical symptomology continued active at the end of the treatment and the TAU did not target well-being or positive 
emotions, suggesting that the improvements in affect and optimistic thinking might be due to the program. In any case, future studies should explore if the combination of this group program with the Treatment as Usual (TAU) produce larger benefits in positive functioning variables, compared to patients who only receive the TAU. Another limitation is that it was the therapist who asked to complete the measures during the intervention period and this might produce a social desirability bias where patients would tend to answer the acceptability measures in a favorable manner. Future studies should include an independent researcher to collect the measures, in order to prevent social desirability effects. To examine the possible benefits of the intervention, it would be interesting to include other measures of psychological and subjective well-being that could also be enhanced by the program, such as hope, self-efficacy, or life satisfaction.

In conclusion, the potential benefits observed in this study, along with the results obtained in another pilot study [10], open the door to carrying out randomized clinical trials (RCT) with severe populations, such as ED patients, in order to draw firmer conclusions about the real benefits of PPI group interventions in clinical populations. If they are found beneficial in further RCTs, the inclusion of PPIs in the context of mental health services could be considered in order to focus not only on the disease symptomatology, but also on positive aspects of functioning [39]. As noted by different authors, the presence of well-being in individuals with clinical disorders could buffer the detrimental effects of the symptoms [40]. This new approach, might support the efficacy of current treatments, even acting as a complementary objective, in order to improve wellbeing and positive emotions in individuals with eating disorders.

\section{What is already known on this subject?}

Eating disorders produce a strong impact in quality of life and are negatively associated to life satisfaction and wellbeing. Most of treatments for these conditions are focused on 
reducing clinical symptomology, while positive affect and wellbeing promotion remain unaddressed. Some authors claim the need of developing interventions aimed at promoting positive emotions that can complement existing treatments and ameliorate the harmful effects of eating disorder symptomology, but evidence on this field is still scarce.

\section{What this study adds?}

The inpatients of this specialized mental health service found the positive psychological group program and the technology used acceptable and useful. Statistically significant improvements in optimistic thinking were also observed. The results are in line with a similar pilot study and open the door to conduct randomized controlled trials to confirm the benefits of these interventions for promoting positive emotions and wellbeing in individuals with eating disorders.

\section{Compliance with Ethical Standards}

Funding for the study was provided by grants: Red de Excelencia (PSI2014-56303REDT) PROMOSAM: Research in processes, mechanisms and psychological treatments for mental health promotion from the Ministerio de Economía y Competitividad (2014); a PhD grant from Jaume I University (PREDOC/2012/51), and CIBER: CIBER Fisiopatología de la Obesidad y Nutrición is an initiative of ISCIII.

All the authors declare that they have no conflict of interest.

We confirm that any aspect of the work covered in this manuscript that has involved human patients has been conducted with the ethical approval of all relevant bodies and that such approvals are acknowledged within the manuscript. The study was approved by the Research Ethics Committee of the Provincial Hospital of Castellón (Spain; October 31st, 2014). All the patients signed an informed consent form, and in the case of underage patients, this consent form was signed by their parents.

\section{References}


1. Arcelus J, Mitchell AJ, Wales J, Nielsen S (2011) Mortality Rates in Patients With Anorexia Nervosa and Other Eating Disorders. Arch Gen Psychiatry 68:724-731. https://doi.org/10.1001/archgenpsychiatry.2011.74

2. Watson HJ, Bulik CM (2013) Update on the treatment of anorexia nervosa: review of clinical trials, practice guidelines and emerging interventions. Psychol Med 43:2477-500. https://doi.org/10.1017/S0033291712002620

3. Fairburn CG (2005) Evidence-based treatment of anorexia nervosa. Int J Eat Disord 37:S26-S30. https://doi.org/10.1002/eat.20112

4. Wonderlich S, Mitchell JE, Crosby RD, et al (2012) Minimizing and treating chronicity in the eating disorders: A clinical overview. Int J Eat Disord 45:467475. https://doi.org/10.1002/eat.20978

5. Jenkins PE, Hoste RR, Meyer C, Blissett JM (2011) Eating disorders and quality of life: A review of the literature. Clin Psychol Rev 31:113-121. https://doi.org/10.1016/j.cpr.2010.08.003

6. Brannan ME, Petrie TA (2011) Psychological well-being and the body dissatisfaction-bulimic symptomatology relationship: An examination of moderators. Eat Behav 12:233-241. https://doi.org/10.1016/j.eatbeh.2011.06.002

7. Kitsantas A, Gilligan TD, Kamata A (2003) College women with eating disorders: self-regulation, life satisfaction, and positive/negative affect. J Psychol 137:381-395. https://doi.org/10.1080/00223980309600622

8. Tozzi F, Sullivan PF, Fear JL, et al (2003) Causes and recovery in anorexia nervosa: The patient's perspective. Int J Eat Disord 33:143-154. https://doi.org/10.1002/eat.10120

9. Tchanturia K, Dapelo MAM, Harrison A, Hambrook D (2015) Why Study Positive Emotions in the Context of Eating Disorders? Curr Psychiatry Rep 17:. 
https://doi.org/10.1007/s11920-014-0537-x

10. Harrison A, Al-Khairulla H, Kikoler M (2015) The feasibility, acceptability and possible benefit of a positive psychology intervention group in an adolescent inpatient eating disorder service. J Posit Psychol Feb:1-11. https://doi.org/10.1080/17439760.2015.1117125

11. Lyubomirsky S, Layous K (2013) How Do Simple Positive Activities Increase Well-Being? Curr Dir Psychol Sci 22:57-62. https://doi.org/10.1177/0963721412469809

12. Malson H, Lin B, Clarke S, et al (2011) Un/imaginable future selves: A discourse analysis of in-patients' talk about recovery from an "eating disorder." Eur Eat Disord Rev 19:25-36. https://doi.org/10.1002/erv.1011

13. Turton P, Demetriou A, Boland W, et al (2011) One size fits all: Or horses for courses? Recovery-based care in specialist mental health services. Soc Psychiatry Psychiatr Epidemiol 46:127-136. https://doi.org/10.1007/s00127-009-0174-6

14. Malouff JM, Schutte NS (2016) Can psychological interventions increase optimism? A meta-analysis. J Posit Psychol Aug:1-11. https://doi.org/10.1080/17439760.2016.1221122

15. King L a. (2001) The Health Benefits of Writing about Life Goals. Personal Soc Psychol Bull 27:798-807. https://doi.org/10.1177/0146167201277003

16. Leon AC, Davis LL, Kraemer HC (2012) Role and Interpretation of Pilot Studies in Clinical Research. J Psychiatr Res 45:626-629. https://doi.org/10.1016/j.jpsychires.2010.10.008.The

17. APA (2000) DSM-IV-TR: Diagnostic and statistical manual of mental disorders, text revision. Washington, DC

18. Baños RM, Etchemendy E, Farfallini L, et al (2014) EARTH of Well-Being 
System: A pilot study of an information and communication technology-based positive psychology intervention. J Posit Psychol 9:482-488. https://doi.org/10.1080/17439760.2014.927906

19. Botella C, Baños RM, Etchemendy E, et al (2016) Psychological countermeasures in manned space missions:"EARTH” system for the Mars-500 project. Comput Human Behav 55:898-908. https://doi.org/https://doi.org/10.1016/j.chb.2015.10.010

20. MacLeod AK (1996) Affect, emotional disorder, and future-directed thinking. Cogn Emot 10:69-86. https://doi.org/https://doi.org/10.1080/026999396380394

21. Meevissen YMC, Peters ML, Alberts HJEM (2011) Become more optimistic by imagining a best possible self: effects of a two week intervention. J Behav Ther Exp Psychiatry 42:371-8. https://doi.org/10.1016/j.jbtep.2011.02.012

22. Enrique A, Bretón-López J, Molinari G, et al (2018) Implementation of a positive technology application in patients with eating disorders: A pilot randomized control trial. Front Psychol 9:1-13. https://doi.org/10.3389/fpsyg.2018.00934

23. Watson D, Clark LA, Tellegen A (1988) Development and validation of brief measures of positive and negative affect: the PANAS scales. J Pers Soc Psychol $54: 1063$

24. Sandín B, Chorot P, Lostao L, et al (1999) Escalas PANAS de afecto positivo y negativo: validación factorial y convergencia transcultural. Psicothema 11:37-51

25. Borkovec TD, Nau SD (1972) Credibility of analogue therapy rationales. J Behav Ther Exp Psychiatry 3:257-260. https://doi.org/10.1016/0005-7916(72)90045-6

26. Garner DM, Olmsted MP, Bohr Y, Garfinkel PE (1982) The eating attitudes test: psychometric features and clinical correlates. Psychol Med 12:871-878. https://doi.org/https://doi.org/10.1017/S0033291700049163 
27. Toro J, Castro J, Garcia M, et al (1989) Eating attitudes, sociodemographic factors and body shape evaluation in adolescence. Br J Med Psychol 62:61-70. https://doi.org/https://doi.org/10.1111/j.2044-8341.1989.tb02811.x

28. Rivas T, Bersabé R, Jiménez M, Berrocal C (2010) The Eating Attitudes Test (EAT-26): Reliability and Validity in Spanish Female Samples. Span J Psychol 13:1044-1056. https://doi.org/10.1017/S1138741600002687

29. Solano N, Cano A (2010) IMAGEN Evaluación de la Insatisfacción de la Imagen Corporal. TEA Ediciones, Madrid

30. Snyder CR (2000) Handbook of hope : theory, measures \& applications. Academic Press, Kansas, USA

31. Jacobson NS, Truax P (1991) Clinical Significance: A Statistical Approach to Defining Meaningful Change in Psychotherapy Research. J Consult Clin Psychol 59:12-19. https://doi.org/10.1037/0022-006X.59.1.12

32. Dragomir-Davis A. (2014) Future-Directed Thinking in General and Clinical Samples: Validation of the Spanish Version of the Subjective Probability Task. Universitat Jaume I

33. DeJong H, Oldershaw A, Sternheim L, et al (2013) Quality of life in anorexia nervosa, bulimia nervosa and eating disorder not-otherwise-specified. J Eat Disord 1:1-8. https://doi.org/10.1186/2050-2974-1-43

34. de Graaf LE, Huibers MJH, Riper H, et al (2009) Use and acceptability of unsupported online computerized cognitive behavioral therapy for depression and associations with clinical outcome. J Affect Disord 116:227-231. https://doi.org/10.1016/j.jad.2008.12.009

35. Park J, Chen RK (2007) Positive Psychology and Hope as Means to Recovery from Mental Illness. J Appl Rehabil Couns 47:34-43. 
https://doi.org/10.1891/0047-2220.47.2.34

36. Hayes SC, Hayes SC, Luoma JB, et al (2006) Acceptance and commitment therapy: model, processes and outcomes. Behav Res Ther 44:1-25. https://doi.org/10.1016/j.brat.2005.06.006

37. King LA, Raspin C (2004) Lost and Found Possible Selves, Subjective WellBeing, and Ego Development in Divorced Women. J Pers 72:603-632. https://doi.org/https://doi.org/10.1111/j.0022-3506.2004.00274.x

38. Botella C, Riva G, Gaggioli A, et al (2012) The present and future of positive technologies. Cyberpsychol Behav Soc Netw 15:78-84.

https://doi.org/10.1089/cyber.2011.0140

39. Tomba E, Tecuta L, Schumann R, Ballardini D (2017) Does psychological wellbeing change following treatment? An exploratory study on outpatients with eating disorders. Compr Psychiatry 74:61-69. https://doi.org/10.1016/j.comppsych.2017.01.001

40. Bos EH, Snippe E, de Jonge P, Jeronimus BF (2016) Preserving Subjective Wellbeing in the Face of Psychopathology: Buffering Effects of Personal Strengths and Resources. PLoS One 11:e0150867. https://doi.org/10.1371/journal.pone.0150867 